\title{
A New Variable-Coefficient Riccati Subequation Method for Solving Nonlinear Lattice Equations
}

\author{
Fanwei Meng \\ School of Mathematical Sciences, Qufu Normal University, Qufu 273165, China \\ Correspondence should be addressed to Fanwei Meng; fengqinghua1978@126.com
}

Received 20 January 2013; Accepted 31 January 2013

Academic Editor: Andrei Korobeinikov

Copyright (C) 2013 Fanwei Meng. This is an open access article distributed under the Creative Commons Attribution License, which permits unrestricted use, distribution, and reproduction in any medium, provided the original work is properly cited.

We propose a new variable-coefficient Riccati subequation method to establish new exact solutions for nonlinear differentialdifference equations. For illustrating the validity of this method, we apply it to the discrete $(2+1)$-dimensional Toda lattice equation. As a result, some new and generalized traveling wave solutions including hyperbolic function solutions, trigonometric function solutions, and rational function solutions are obtained.

\section{Introduction}

Nonlinear differential-difference equations (NDDEs) play an important role in many branches of applied physical sciences such as condensed matter physics, biophysics, atomic chains, molecular crystals, and quantum physics. Unlike difference equations which are fully discretized, NDDEs are semidiscretized; that is, their time variable is usually kept continuous. Since the work of Fermi in the 1960s [1], research for NDDEs has been paid a lot of attention recently (e.g., see [2-10] and the references therein). Among these research works, the investigation of exact solutions of NDDEs has attracted a wide interest, and many effective methods have been presented and applied for solving NDDEs successfully in the literature. For example, these methods include the known $\left(G^{\prime} / G\right)$-expansion method [11-14], the exp-function method [15], the exponential function rational expansion method $[16,17]$, the Jacobi elliptic function method $[18,19]$, Hirota's bilinear method [20], the extended simplest equation method [21], and the tanh-function method [22]. The difference among these methods lies in that different ansatzes are used. However, we notice most of the existing methods are dealing with ansatzes with constant coefficients, while very few methods are concerned with variable coefficients. Recent results on solving NDDEs by variable-coefficient methods only include the presentation of Tang et al. [14], in which a variable-coefficient $\left(G^{\prime} / G\right)$-expansion method was developed.

It is well known that the Riccati equation method is very effective in finding exact solutions for nonlinear differentialdifference equations [23,24]. But so far little attention is paid to the application of the Riccati equation method to solve NDDEs. In this paper, by introducing a new ansatz, we propose a new discrete variable-coefficient Riccati subequation method for solving NDDEs. Then we apply this method to establish new exact solutions of NDDEs.

We organize this paper as follows. In Section 2, we give the description of the discrete variable-coefficient Riccati subequation method. Then in Section 3 we apply the method to solve the discrete $(2+1)$-dimensional Toda lattice equation. Comparisons between the proposed method and the known $\left(G^{\prime} / G\right)$-expansion method are also made. Some conclusions are presented at the end of the paper.

\section{Description of the Variable-Coefficient Riccati Subequation Method}

The main steps of the variable-coefficient Riccati sub-ODE method for solving NDDEs are summarized as follows. 
Step 1. Consider a system of $M$ polynomial NDDEs in the form

$$
\begin{gathered}
P\left(u_{n+p_{1}}(X), \ldots, u_{n+p_{k}}(X), \ldots,\right. \\
u_{n+p_{1}}^{\prime}(X), \ldots, u_{n+p_{k}}^{\prime}(X), \ldots, \\
\left.u_{n+p_{1}}^{(r)}(X), \ldots, u_{n+p_{k}}^{(r)}(X)\right)=0,
\end{gathered}
$$

where the dependent variable $u$ has $M$ components $u_{i}$, the continuous variable $X$ has $N$ components $x_{j}$, the discrete variable $n$ has $Q$ components $n_{i}$, the $k$ shift vectors $p_{s} \in Z^{Q}$ have $Q$ components $p_{s j}$, and $u_{n+p_{s}}^{(r)}(X), s=1,2, \ldots, k$, denote the collection of mixed derivative terms of order $r$.

Step 2. Using a wave transformation

$$
\begin{gathered}
u_{n+p_{s}}(X)=U_{n+p_{s}}\left(\xi_{n+p_{s}}\right), \\
\xi_{n}=\sum_{i=1}^{Q} d_{i} n_{i}+\zeta(X), \\
\xi_{n+p_{s}}=\sum_{i=1}^{Q} d_{i}\left(n_{i}+p_{s i}\right)+\zeta(X),
\end{gathered}
$$

where $d_{i}, p_{s i}, i=1, \ldots, Q$, are constants and $\zeta(X)$ are underdetermined differentiable functions of $X$, we can rewrite (1) in the following nonlinear ODE:

$$
\begin{aligned}
\widetilde{P}( & U_{n+p_{1}}\left(\xi_{n+p_{1}}\right), \ldots, \\
& U_{n+p_{k}}\left(\xi_{n+p_{k}}\right), \ldots, U_{n+p_{1}}^{\prime}\left(\xi_{n+p_{1}}\right), \ldots, \\
& U_{n+p_{k}}^{\prime}\left(\xi_{n+p_{k}}\right), \ldots, \\
& \left.U_{n+p_{1}}^{(r)}\left(\xi_{n+p_{1}}\right), \ldots, U_{n+p_{k}}^{(r)}\left(\xi_{n+p_{k}}\right)\right)=0 .
\end{aligned}
$$

Step 3. Suppose the solutions of (3) can be denoted by

$$
U_{n}\left(\xi_{n}\right)=\sum_{i=0}^{l} a_{i}(X) \phi^{i}\left(\xi_{n}\right),
$$

where $a_{i}(X)$ are functions of $X$ to be determined later, $l$ is a positive integer that can be determined by balancing the highest order linear term with the nonlinear terms in (3), and $\phi\left(\xi_{n}\right)$ satisfies the known Riccati equation:

$$
\phi^{\prime}\left(\xi_{n}\right)=\sigma+\phi^{2}\left(\xi_{n}\right) .
$$

Step 4. We present some special solutions $\phi_{1}, \ldots \phi_{6}$ for (5).

When $\sigma<0$,

$$
\begin{aligned}
& \phi_{1}\left(\xi_{n}\right)=-\sqrt{-\sigma} \tanh \left(\sqrt{-\sigma} \xi_{n}+c_{0}\right), \\
& \phi_{2}\left(\xi_{n}\right)=-\sqrt{-\sigma} \operatorname{coth}\left(\sqrt{-\sigma} \xi_{n}+c_{0}\right), \\
& \phi_{1,2}\left(\xi_{n+p_{s}}\right)=\frac{\phi_{1,2}\left(\xi_{n}\right)-\sqrt{-\sigma} \tanh \left(\sqrt{-\sigma} \sum_{i=1}^{\mathrm{Q}} d_{i} p_{s i}\right)}{1-\left(\phi_{1,2}\left(\xi_{n}\right) / \sqrt{-\sigma}\right) \tanh \left(\sqrt{-\sigma} \sum_{i=1}^{\mathrm{Q}} d_{i} p_{s i}\right)},
\end{aligned}
$$

where $c_{0}$ is an arbitrary constant.
When $\sigma>0$,

$$
\begin{aligned}
& \phi_{3}\left(\xi_{n}\right)=\sqrt{\sigma} \tan \left(\sqrt{\sigma} \xi_{n}+c_{0}\right) \\
& \phi_{4}\left(\xi_{n}\right)=-\sqrt{\sigma} \cot \left(\sqrt{\sigma} \xi_{n}+c_{0}\right) \\
& \phi_{3,4}\left(\xi_{n+p_{s}}\right) \\
& =\frac{\phi_{3,4}\left(\xi_{n}\right)+\sqrt{\sigma} \tan \left(\sqrt{\sigma} \sum_{i=1}^{Q} d_{i} p_{s i}\right)}{1-\left(\phi_{3,4}\left(\xi_{n}\right) / \sqrt{\sigma}\right) \tan \left(\sqrt{\sigma} \sum_{i=1}^{\mathrm{Q}} d_{i} p_{s i}\right)}, \\
& \phi_{5}\left(\xi_{n}\right)=\sqrt{\sigma}\left[\tan \left(2 \sqrt{\sigma} \xi_{n}+c_{0}\right)\right. \\
& \left.\phi_{5}\left(\xi_{n+p_{s}}\right) \quad+\left|\sec \left(2 \sqrt{\sigma} \xi_{n}+c_{0}\right)\right|\right], \\
& =\frac{\phi_{5}^{(1)}\left(\xi_{n}\right)+\sqrt{\sigma} \tan \left(2 \sqrt{\sigma} \sum_{i=1}^{Q} d_{i} p_{s i}\right)}{1-\left(\phi_{5}^{(1)}\left(\xi_{n}\right) / \sqrt{\sigma}\right) \tan \left(2 \sqrt{\sigma} \sum_{i=1}^{\mathrm{Q}} d_{i} p_{s i}\right)} \\
& \quad+\frac{\phi_{5}^{(2)}\left(\xi_{n}\right) \sec \left(2 \sqrt{\sigma} \sum_{i=1}^{\mathrm{Q}} d_{i} p_{s i}\right)}{1-\left(\phi_{5}^{(1)}\left(\xi_{n}\right) / \sqrt{\sigma}\right) \tan \left(2 \sqrt{\sigma} \sum_{i=1}^{\mathrm{Q}} d_{i} p_{s i}\right)}
\end{aligned}
$$

where $\phi_{5}^{(1)}\left(\xi_{n}\right)=\sqrt{\sigma} \tan \left(2 \sqrt{\sigma} \xi_{n}+c_{0}\right), \phi_{5}^{(2)}\left(\xi_{n}\right)=$ $\sqrt{\sigma} \mid \sec \left(2 \sqrt{\sigma} \xi_{n}+c_{0}\right)$ and $c_{0}$ is an arbitrary constant.

When $\sigma=0$,

$$
\begin{gathered}
\phi_{6}\left(\xi_{n}\right)=-\frac{1}{\xi_{n}+c_{0}}, \\
\phi_{6}\left(\xi_{n+p_{s}}\right)=\frac{\phi_{6}\left(\xi_{n}\right)}{1-\phi_{6}\left(\xi_{n}\right) \sum_{i=1}^{Q} d_{i} p_{s i}}
\end{gathered}
$$

where $c_{0}$ is an arbitrary constant.

Step 5. Substituting (4) into (3), by use of (5)-(9), the left hand side of (3) can be converted into a polynomial in $\phi\left(\xi_{n}\right)$. Equating each coefficient of $\phi^{i}\left(\xi_{n}\right)$ to zero, yields a set of algebraic equations. Solving these equations, we can obtain a set of under-determined partial equations, from which $a_{i}(X), \zeta(X)$ can be determined using the aid of mathematical software.

Step 6. Substituting the values of $a_{i}(X)$ into (4) and combining with the various solutions of (5), we can obtain a variety of exact solutions for (1).

Remark 1. In [25, equations (40)-(42)], Ma and Fuchssteiner presented a general class of solutions to the following Riccati equation:

$$
v_{\xi}=a_{0}+a_{1} v+a_{2} v^{2}
$$

We note that despite the difference in forms and parameters, our solutions $\phi_{1}\left(\xi_{n}\right), \phi_{2}\left(\xi_{n}\right), \phi_{3}\left(\xi_{n}\right), \phi_{4}\left(\xi_{n}\right)$, and $\phi_{6}\left(\xi_{n}\right)$ to (5) are in accordance with the presented solutions in [25]. In fact, $\phi_{1}\left(\xi_{n}\right)$ is essentially equivalent to the tanh-function solution in $[25,(41)]$ with $a_{1}=0, a_{2}=1, \Delta=-4 \sigma$, and $-\varepsilon \ln \varepsilon_{0} / 2=c_{0}$, while $\phi_{2}\left(\xi_{n}\right)$ is essentially equivalent to the 
coth-function solution in [25, (41)] with $a_{1}=0, a_{2}=1, \Delta=$ $-4 \sigma$, and $-\varepsilon \ln \left(-\varepsilon_{0}\right) / 2=c_{0} . \phi_{3}\left(\xi_{n}\right)$ is essentially equivalent to the tan-function solution in $[25,(42)]$ with $a_{1}=0, a_{2}=1$, $\Delta=-4 \sigma$, and $\xi_{0}=c_{0}$, while $\phi_{4}\left(\xi_{n}\right)$ is essentially equivalent to the cot-function solution in $[25,(42)]$ with $a_{1}=0, a_{2}=1$, $\Delta=-4 \sigma$, and $\xi_{0}=c_{0} \cdot \phi_{7}\left(\xi_{n}\right)$ is essentially equivalent to the rational function solution in [25, (40)] with $a_{1}=0, a_{2}=1$, and $\xi_{0}=c_{0}$. Besides, our solution $\phi_{5}\left(\xi_{n}\right)$ is not shown in [25].

\section{Application of the Variable-Coefficient Riccati Subequation Method to the Discrete $(2+1)$-Dimensional Toda Lattice Equation}

In this section, we will apply the described method in Section 2 to the discrete $(2+1)$-dimensional Toda lattice equation $[13,14]$ :

$$
\frac{\partial^{2} y_{n}}{\partial x \partial t}=e^{y_{n-1}-y_{n}}-e^{y_{n}-y_{n+1}}
$$

where $y_{n}=y_{n}(x, t), n \in Z$, is the displacement from equilibrium of the $n$th unit mass under an exponentially decaying interaction force between nearest neighbors.

In [26], Ma presented a general Casoratian formulation for the 2D Toda lattice equation and obtained various Casoratian-type solutions by a Bäcklund transformation under some linear conditions. In [27], Ma derived molecule solutions in Wronskian form for the finite, semi-infinite, and infinite bilinear 2D Toda molecule equations by combining two pieces of molecule bidirectional Wronskian solutions, and in the cases of finite and semi-infinite lattices, separatedvariable boundary conditions are imposed.

As in $[13,14]$, using the transformation

$$
\frac{\partial u_{n}}{\partial t}=e^{y_{n-1}-y_{n}}-1
$$

(11) can be rewritten as

$$
\frac{\partial^{2} u_{n}}{\partial x \partial t}=\left(\frac{\partial u_{n}}{\partial t}+1\right)\left(u_{n-1}-2 u_{n}+u_{n+1}\right)
$$

Using a wave transformation

$$
u_{n}(x, t)=U_{n}\left(\xi_{n}\right), \quad \xi_{n}=d_{1} n+\zeta(x, t),
$$

where $d_{1}$ is a constant, (13) can be turned into

$$
\begin{aligned}
& \frac{\partial \zeta(x, t)}{\partial x} \frac{\partial \zeta(x, t)}{\partial t}+U_{n}^{\prime \prime} \frac{\partial^{2} \zeta(x, t)}{\partial x \partial t} U_{n}^{\prime} \\
& \quad=\left(\frac{\partial \zeta(x, t)}{\partial t} U_{n}^{\prime}+1\right)\left(U_{n-1}-2 U_{n}+U_{n+1}\right),
\end{aligned}
$$

where $U_{n}=U_{n}\left(\xi_{n}\right), U_{n}^{\prime}=U_{n}^{\prime}\left(\xi_{n}\right)$. Suppose $U_{n}\left(\xi_{n}\right)$ can be denoted by

$$
U_{n}\left(\xi_{n}\right)=\sum_{i=0}^{l} a_{i}(x, t) \phi^{i}\left(\xi_{n}\right),
$$

where $\phi\left(\xi_{n}\right)$ satisfies (5). Balancing the order of $U_{n}^{\prime \prime}$ and $U_{n}^{\prime} U_{n}$ in (15), we obtain $l+2=(l+1)+l$, and then $l=1$. So we have

$$
U_{n}\left(\xi_{n}\right)=a_{0}(x, t)+a_{1}(x, t) \phi\left(\xi_{n}\right) .
$$

Next we will proceed to solve (15) and (13) in several cases.

Case 1. If $\sigma<0$ and assume (5) and (6) hold, then substituting (17), (5), and (6) into (15), collecting the coefficients of $\phi_{1,2}^{i}\left(\xi_{n}\right)$, and equating them to zero, we obtain a series of underdetermined partial equations for $a_{0}(x, t), a_{1}(x, t)$, and $\zeta(x, t)$. Solving these equations yields

$$
\begin{gathered}
a_{1}(x, t)=F_{1}(x), \quad a_{0}=-t+F_{2}(x), \\
\zeta(x, t)=F_{3}(x),
\end{gathered}
$$

where $F_{i}(x), i=1,2,3$, are arbitrary functions with respect to the variable $x$, or

$$
\begin{gathered}
a_{1}(x, t)=-k, \\
a_{0}=-t-\frac{2 k \sigma g_{1}(t)}{-1+\cosh \left(2 \sqrt{-\sigma} d_{1}\right)}+g_{2}(x), \\
\zeta(x, t)=k x+g_{1}(t),
\end{gathered}
$$

where $k$ is an arbitrary nonzero constant and $g_{1}(t)$ and $g_{2}(x)$ are two arbitrary functions with respect to the variables $x$ and $t$, respectively. Then we obtain the following hyperbolic function solitary wave solutions for (13):

$$
\begin{aligned}
u_{n}(x, t)= & -F_{1}(x) \sqrt{-\sigma} \tanh \left(\sqrt{-\sigma}\left(d_{1} n+F_{3}(x)\right)+c_{0}\right) \\
& -t+F_{2}(x)
\end{aligned}
$$

$$
\begin{gathered}
u_{n}(x, t)=-F_{1}(x) \sqrt{-\sigma} \operatorname{coth}\left(\sqrt{-\sigma}\left(d_{1} n+F_{3}(x)\right)+c_{0}\right) \\
-t+F_{2}(x), \\
u_{n}(x, t)=k \sqrt{-\sigma} \tanh \left(\sqrt{-\sigma}\left(d_{1} n+k x+g_{1}(t)\right)+c_{0}\right) \\
-t-\frac{2 k \sigma g_{1}(t)}{-1+\cosh \left(2 \sqrt{-\sigma} d_{1}\right)}+g_{2}(x), \\
u_{n}(x, t)=k \sqrt{-\sigma} \operatorname{coth}\left(\sqrt{-\sigma}\left(d_{1} n+k x+g_{1}(t)\right)+c_{0}\right) \\
-t-\frac{2 k \sigma g_{1}(t)}{-1+\cosh \left(2 \sqrt{-\sigma} d_{1}\right)}+g_{2}(x),
\end{gathered}
$$

where $d_{1}, c_{0}$ are arbitrary constants.

Case 2. If $\sigma>0$ and assume (5) and (7) hold, then substituting (17), (5), and (7) into (15), collecting the coefficients of $\phi_{3,4}^{i}\left(\xi_{n}\right)$, and equating them to zero, we obtain a series of 
underdetermined partial equations for $a_{0}(x, t), a_{1}(x, t)$, and $\zeta(x, t)$. Solving these equations yields

$$
\begin{gathered}
a_{1}(x, t)=F_{1}(x), \quad a_{0}=-t+F_{2}(x), \\
\zeta(x, t)=F_{3}(x)
\end{gathered}
$$

or

$$
\begin{gathered}
a_{1}(x, t)=-k, \\
a_{0}=-t-\frac{2 k \sigma g_{1}(t)}{-1+\cos \left(2 \sqrt{\sigma} d_{1}\right)}+g_{2}(x), \\
\zeta(x, t)=k x+g_{1}(t) .
\end{gathered}
$$

Then we obtain the following trigonometric function solutions for (13):

$$
\begin{aligned}
u_{n}(x, t)= & F_{1}(x) \sqrt{\sigma} \tan \left(\sqrt{\sigma}\left(d_{1} n+F_{3}(x)\right)+c_{0}\right) \\
& -t+F_{2}(x), \\
u_{n}(x, t)=- & F_{1}(x) \sqrt{\sigma} \cot \left(\sqrt{\sigma}\left(d_{1} n+F_{3}(x)\right)+c_{0}\right) \\
& -t+F_{2}(x), \\
u_{n}(x, t)=- & k \sqrt{\sigma} \tan \left(\sqrt{\sigma}\left(d_{1} n+k x+g_{1}(t)\right)+c_{0}\right) \\
& -t-\frac{2 k \sigma g_{1}(t)}{-1+\cos \left(2 \sqrt{\sigma} d_{1}\right)}+g_{2}(x), \\
u_{n}(x, t)= & k \sqrt{\sigma} \cot \left(\sqrt{\sigma}\left(d_{1} n+k x+g_{1}(t)\right)+c_{0}\right) \\
& -t-\frac{2 k \sigma g_{1}(t)}{-1+\cos \left(2 \sqrt{\sigma} d_{1}\right)}+g_{2}(x),
\end{aligned}
$$

where $d_{1}, c_{0}$ are arbitrary constants.

Case 3. If $\sigma>0$ and assume (5) and (8) hold, then substituting (17), (5), and (8) into (15), using $\left[\phi_{5}^{(2)}\left(\xi_{n}\right)\right]^{2}=\sigma+$ $\left[\phi_{5}^{(1)}\left(\xi_{n}\right)\right]^{2}$, collecting the coefficients of $\left[\phi_{5}^{(1)}\left(\xi_{n}\right)\right]^{i}\left[\phi_{5}^{(2)}\left(\xi_{n}\right)\right]^{j}$, and equating them to zero, we obtain a series of partial equations. Solving these equations, we also obtain, as in Case 2 ,

$$
\begin{gathered}
a_{1}(x, t)=F_{1}(x), \quad a_{0}=-t+F_{2}(x), \\
\zeta(x, t)=F_{3}(x)
\end{gathered}
$$

or

$$
\begin{gathered}
a_{1}(x, t)=-k, \\
a_{0}=-t-\frac{2 k \sigma g_{1}(t)}{-1+\cos \left(2 \sqrt{\sigma} d_{1}\right)}+g_{2}(x), \\
\zeta(x, t)=k x+g_{1}(t) .
\end{gathered}
$$

So we have the following trigonometric function solutions for (13):

$$
\begin{aligned}
u_{n}(x, t)=F_{1}(x) \sqrt{\sigma}[ & \tan \left(2 \sqrt{\sigma}\left(d_{1} n+F_{3}(x)\right)+c_{0}\right) \\
+ & \left.\left|\sec \left(2 \sqrt{\sigma}\left(d_{1} n+F_{3}(x)\right)+c_{0}\right)\right|\right] \\
- & t+F_{2}(x),
\end{aligned}
$$

$$
\begin{aligned}
u_{n}(x, t)=-k \sqrt{\sigma}[ & \tan \left(2 \sqrt{\sigma}\left(d_{1} n+k x+g_{1}(t)\right)+c_{0}\right) \\
& \left.+\left|\sec \left(2 \sqrt{\sigma}\left(d_{1} n+k x+g_{1}(t)\right)+c_{0}\right)\right|\right] \\
& -t-\frac{2 k \sigma g_{1}(t)}{-1+\cos \left(2 \sqrt{\sigma} d_{1}\right)}+g_{2}(x),
\end{aligned}
$$

where $d_{1}, c_{0}$ are arbitrary constants.

Case 4. If $\sigma=0$ and assume (5) and (9) hold, then substituting (17), (5), and (9) into (15), collecting the coefficients of $\phi_{6}^{i}\left(\xi_{n}\right)$, and equating them to zero, we obtain a series of partial equations. Solving these equations yields

$$
\begin{gathered}
a_{1}(x, t)=F_{1}(x), \quad a_{0}=-t+F_{2}(x), \\
\zeta(x, t)=F_{3}(x)
\end{gathered}
$$

or

$$
\begin{array}{r}
a_{1}(x, t)=-k, \quad a_{0}=g_{2}(x)+g_{1}(t), \\
\zeta(x, t)=k x+\frac{d_{1}^{2}\left(t+g_{1}(t)\right)}{k}+m,
\end{array}
$$

where $m$ is an arbitrary constant, and then we can obtain the following rational function solutions for (13):

$$
\begin{aligned}
& u_{n}(x, t)=-\frac{F_{1}(x)}{d_{1} n+F_{3}(x)+c_{0}}-t+F_{2}(x), \\
& u_{n}(x, t) \\
& =\frac{k}{d_{1} n+k x+d_{1}^{2}\left(t+g_{1}(t)\right) / k+m+c_{0}} \\
& \quad+g_{2}(x)+g_{1}(t) .
\end{aligned}
$$

Remark 2. In [14], Tang et al. presented some exact solutions for (11) using the variable-coefficient $\left(G^{\prime} / G\right)$-expansion method. We note that our results (22), (28), and (37) are generalizations of [14, equations (17), (23), (29)]. In fact, if we let in (22) $c_{0}=\operatorname{arth}\left(C_{2} / C_{1}\right)$ or $\operatorname{arcoth}\left(C_{2} / C_{1}\right)$, $\sigma=\left(4 \mu-\lambda^{2}\right) / 4, g_{1}(t)=g(t)$, and $g_{2}(x)=h(x)-k \lambda / 2$, then the solution denoted by (22) becomes [14, equation (17)]. If we let in (28) $c_{0}=\arctan \left(-C_{2} / C_{1}\right)$ or $\operatorname{arccot}\left(-C_{2} / C_{1}\right)$, $\sigma=\left(4 \mu-\lambda^{2}\right) / 4, g_{1}(t)=g(t)$, and $g_{2}(x)=h(x)-k \lambda / 2$, then the solution denoted by (28) becomes [14, equation (23)]. If we let in (37) $c_{0}=C_{2} / C_{1}, g_{1}(t)=k g(t) / d_{1}^{2}-t, m=k$, and $g_{2}(x)=h(x)-k \lambda / 2$, then the solution denoted by (37) becomes [14, equation (29)]. 
Remark 3. It is worthy to note that the solutions in $[14$, equations (18), (24), (30)] are obtained under the hypothesis

$$
U_{n}\left(\xi_{n}\right)=\sum_{l=-m}^{m} a_{i}(X) \frac{G^{\prime}\left(\xi_{n}\right)}{G\left(\xi_{n}\right)} .
$$

If we modify (4) by

$$
U_{n}\left(\xi_{n}\right)=\sum_{i=-l}^{l} a_{i}(X) \phi^{i}\left(\xi_{n}\right),
$$

then following a similar manner as above we can obtain some exact solutions for (13), which are generalizations of [14, equations (18), (24), (30)]. As the process is almost the same, we omit it here.

Remark 4. Our results (32) and (33) are new exact solutions for (13) and have not been reported by other authors so far to our best knowledge.

Remark 5. From the analysis above, we notice that more general exact solutions for the discrete $(2+1)$-dimensional Toda lattice equation are obtained by the proposed variablecoefficient Riccati subequation method than by the $\left(G^{\prime} / G\right)$ expansion method. In fact, in the $\left(G^{\prime} / G\right)$-expansion method, the solutions $U_{n}\left(\xi_{n}\right)$ are denoted by a polynomial in $\left(G^{\prime}\left(\xi_{n}\right) /\right.$ $\left.G\left(\xi_{n}\right)\right)$, and $G$ satisfies

$$
G^{\prime \prime}+\lambda G^{\prime}+\mu G=0,
$$

where $\lambda, \mu$ are constants. If we let in $(40)\left(G^{\prime}\left(\xi_{n}\right) / G\left(\xi_{n}\right)\right)=$ $-\phi\left(\xi_{n}\right)-\lambda / 2,\left(4 \mu-\lambda^{2}\right) / 4=\sigma$, then $(40)$ reduces to $\phi^{\prime}\left(\xi_{n}\right)=$ $\sigma+\phi^{2}\left(\xi_{n}\right)$, which is the Riccati equation (5). So $\left(G^{\prime}\left(\xi_{n}\right) / G\left(\xi_{n}\right)\right)$ can be generalized and explained by $\phi\left(\xi_{n}\right)$.

Remark 6. All of the solutions presented in this paper have been checked with Maple 11 by putting them back into the original equations.

\section{Conclusions}

We have proposed a variable-coefficient Riccati sub-equation method for solving nonlinear differential-difference equations and applied it to find exact solutions of the discrete $(2+1)$-dimensional Toda lattice equation. As a result, some generalized and new exact solutions for it have been successfully found. We have also compared this method with the known $\left(G^{\prime} / G\right)$-expansion method. Comparison results show that solutions obtained by the $\left(G^{\prime} / G\right)$-expansion method are only special cases of the solutions by the proposed method, which is to some extent in accordance with the analysis in [28]. Finally, as the present method is concise and powerful, we note that it can be applied to other nonlinear differentialdifference equations.

\section{Acknowledgments}

This work is partially supported by National Natural Science Foundation of China (11171178). The author would like to thank the reviewers very much for their valuable suggestions on the paper.

\section{References}

[1] E. Fermi, Collected Papers of Enrico Fermi II, The University of Chicago Press, Chicago, Ill, USA, 1965.

[2] M. Wadati, "Transformation theories for nonlinear discrete systems," Progress of Theoretical Physics, vol. 59, pp. 36-63, 1976.

[3] M. K. Kadalbajoo and K. K. Sharma, "Numerical treatment for singularly perturbed nonlinear differential difference equations with negative shift," Nonlinear Analysis, Theory, Methods and Applications, vol. 63, no. 5-7, pp. e1909-e1924, 2005.

[4] M. Toda, Theory of Nonlinear Lattices, vol. 20, Springer, Berlin, Germany, 1981.

[5] D. Baldwin, Ü. Göktaş, and W. Hereman, "Symbolic computation of hyperbolic tangent solutions for nonlinear differentialdifference equations," Computer Physics Communications, vol. 162, no. 3, pp. 203-217, 2004.

[6] M. J. Ablowitz and J. F. Ladik, "Nonlinear differential-difference equations," Journal of Mathematical Physics, vol.16, pp. 598-603, 1975.

[7] S.-K. Liu, Z.-T. Fu, Z.-G. Wang, and S.-D. Liu, "Periodic solutions for a class of nonlinear differential-difference equations," Communications in Theoretical Physics, vol. 49, no. 5, pp. 11551158, 2008.

[8] L. Wu, L.-D. Xie, and J.-F. Zhang, "Adomian decomposition method for nonlinear differential-difference equations," Communications in Nonlinear Science and Numerical Simulation, vol. 14, no. 1, pp. 12-18, 2009.

[9] P. Marquié, J. M. Bilbault, and M. Remoissenet, "Observation of nonlinear localized modes in an electrical lattice," Physical Review E, vol. 51, no. 6, pp. 6127-6133, 1995.

[10] H. S. Eisenberg, Y. Silberberg, R. Morandotti, A. R. Boyd, and J. S. Aitchison, "Discrete spatial optical solitons in waveguide arrays," Physical Review Letters, vol. 81, no. 16, pp. 3383-3386, 1998.

[11] I. Aslan, "Discrete exact solutions to some nonlinear differential-difference equations via the $\left(G^{\prime} / G\right)$-expansion method," Applied Mathematics and Computation, vol. 215, no. 8, pp. 31403147, 2009.

[12] B. Ayhan and A. Bekir, "The $\left(G^{\prime} / G\right)$-expansion method for the nonlinear lattice equations," Communications in Nonlinear Science and Numerical Simulation, vol. 17, pp. 3490-3498, 2012.

[13] S. Zhang, L. Dong, J.-M. Ba, and Y.-N. Sun, “The $\left(G^{\prime} / G\right)$ expansion method for nonlinear differential-difference equations," Physics Letters A, vol. 373, no. 10, pp. 905-910, 2009.

[14] B. Tang, Y. N. He, L. L. Wei, and S. L. Wang, "Variable-coefficient discrete $\left(G^{\prime} / G\right)$-expansion method for nonlinear differentialdifference equations," Physics Letters A, vol. 375, pp. 3355-3361, 2011.

[15] C.-Q. Dai, X. Cen, and S.-S. Wu, "Exact travelling wave solutions of the discrete sine-Gordon equation obtained via the exp-function method," Nonlinear Analysis. Theory, Methods \& Applications A, vol. 70, no. 1, pp. 58-63, 2009.

[16] C.-S. Liu, "Exponential function rational expansion method for nonlinear differential-difference equations," Chaos, Solitons and Fractals, vol. 40, no. 2, pp. 708-716, 2009.

[17] H. Xin, "The exponential function rational expansion method and exact solutions to nonlinear lattice equations system," Applied Mathematics and Computation, vol. 217, no. 4, pp. 15611565, 2010. 
[18] K. A. Gepreel and A. R. Shehata, "Rational Jacobi elliptic solutions for nonlinear differential-difference lattice equations," Applied Mathematics Letters, vol. 25, no. 9, pp. 1173-1178, 2012.

[19] W. Huang and Y. Liu, "Jacobi elliptic function solutions of the Ablowitz-Ladik discrete nonlinear Schrödinger system," Chaos, Solitons and Fractals, vol. 40, no. 2, pp. 786-792, 2009.

[20] X.-B. Hu and W.-X. Ma, "Application of Hirotas bilinear formalism to the Toeplitz lattice-some special soliton-like solutions," Physics Letters A, vol. 293, no. 3-4, pp. 161-165, 2002.

[21] I. Aslan, "A discrete generalization of the extended simplest equation method," Communications in Nonlinear Science and Numerical Simulation, vol. 15, no. 8, pp. 1967-1973, 2010.

[22] Z. Wang, "Discrete tanh method for nonlinear differencedifferential equations," Computer Physics Communications, vol. 180, no. 7, pp. 1104-1108, 2009.

[23] N. A. Kudryashov, "Simplest equation method to look for exact solutions of nonlinear differential equations," Chaos, Solitons and Fractals, vol. 24, no. 5, pp. 1217-1231, 2005.

[24] N. A. Kudryashov, "Exact solitary waves of the Fisher equation," Physics Letters A, vol. 342, no. 1-2, pp. 99-106, 2005.

[25] W. X. Ma and B. Fuchssteiner, "Explicit and exact solutions to a Kolmogorov-Petrovskii-Piskunov equation," International Journal of Non-Linear Mechanics, vol. 31, no. 3, pp. 329-338, 1996.

[26] W. X. Ma, "An application of the Casoratian technique to the 2D Toda lattice equation," Modern Physics Letters B, vol. 22, no. 19, pp. 1815-1825, 2008.

[27] W.-X. Ma, "Combined Wronskian solutions to the 2D Toda molecule equation," Physics Letters A, vol. 375, no. 45, pp. 39313935, 2011.

[28] N. A. Kudryashov, "A note on the $\left(G^{\prime} / G\right)$-expansion method," Applied Mathematics and Computation, vol. 217, no. 4, pp. 17551758, 2010. 


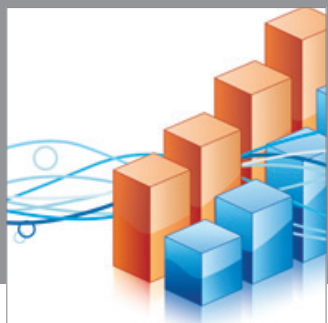

Advances in

Operations Research

mansans

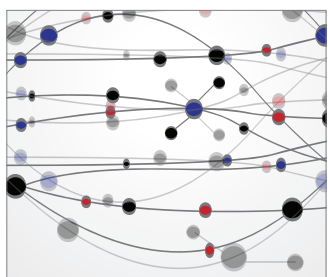

The Scientific World Journal
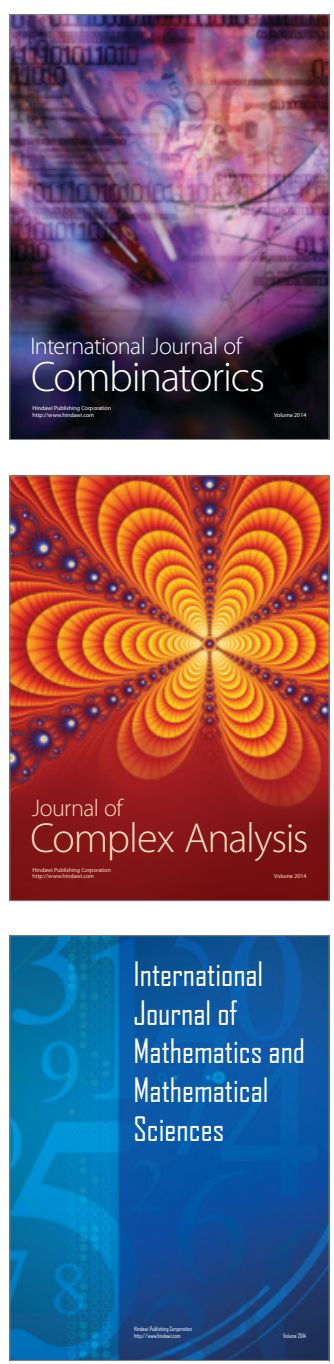
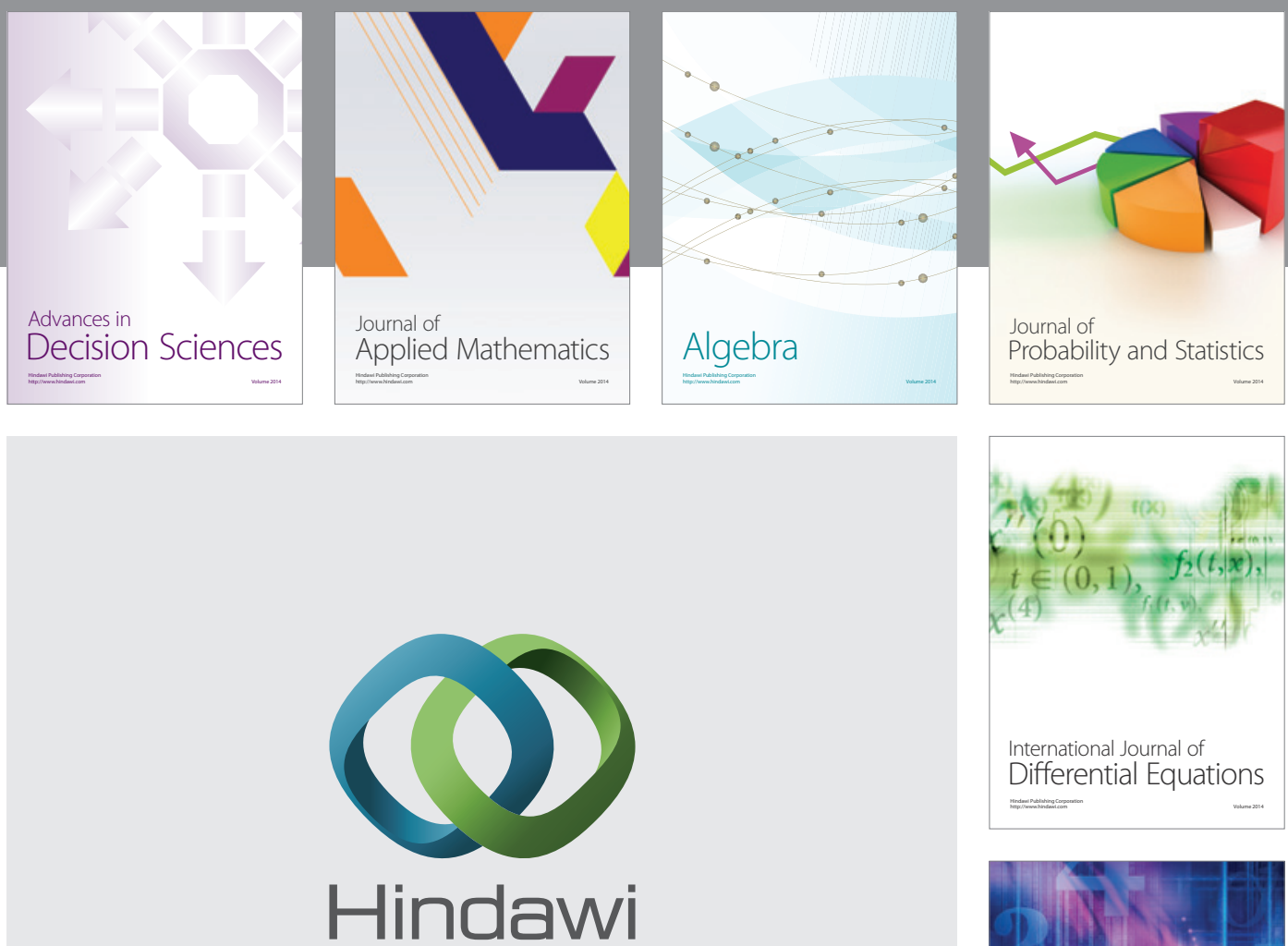

Submit your manuscripts at http://www.hindawi.com
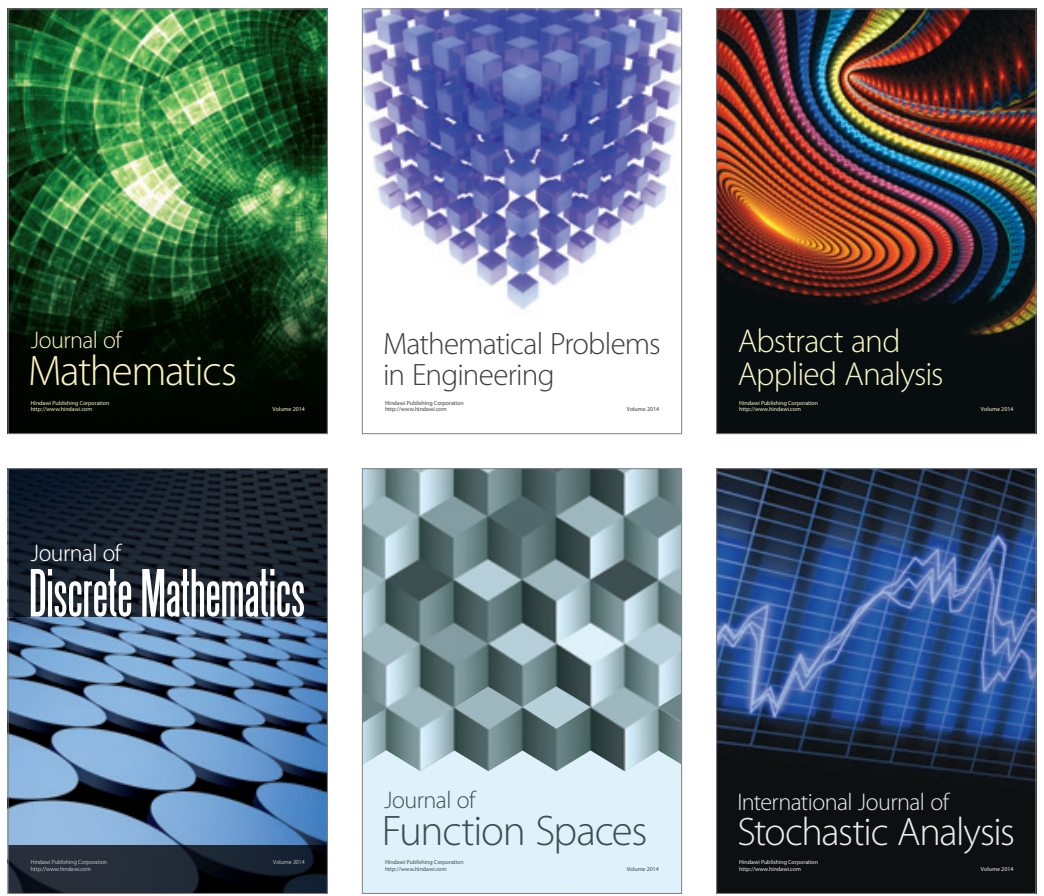

Journal of

Function Spaces

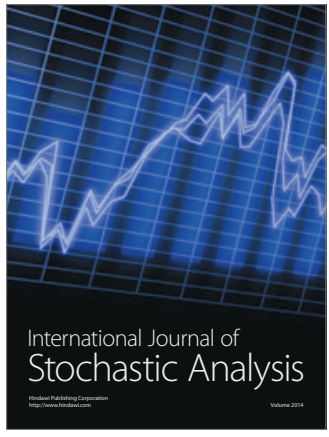

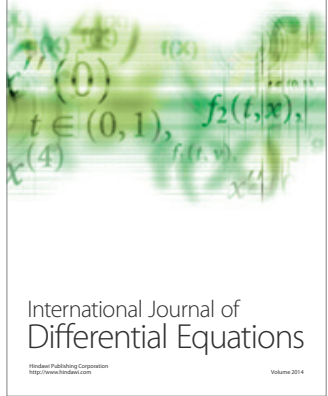
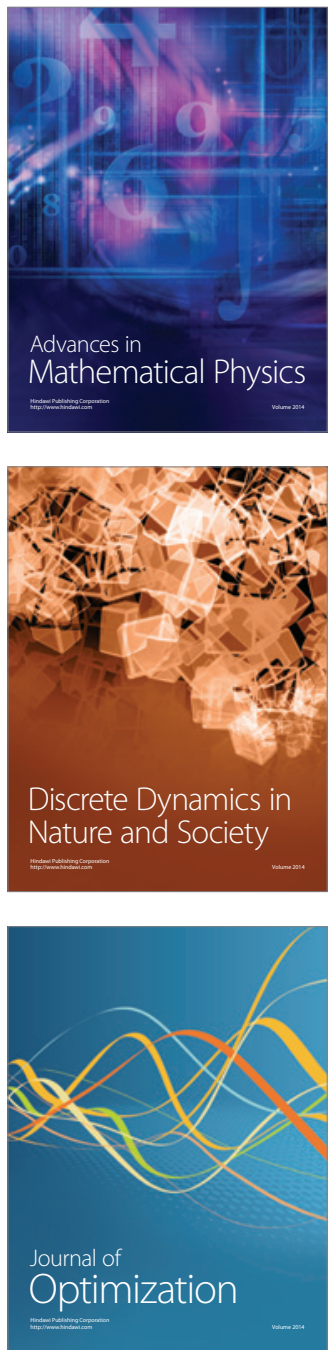